\title{
Solution and crystal structures of a C-terminal fragment of the neuronal isoform of the polypyrimidine tract binding protein (nPTB)
}

The eukaryotic polypyrimidine tract binding protein (PTB) serves primarily as a regulator of alternative splicing of messenger RNA, but is also co-opted to other roles such as RNA localisation and translation initiation from internal ribosome entry sites. The neuronal paralogue of PTB (nPTB) protein is $75 \%$ identical in amino acid sequence with PTB. Although the two proteins have broadly similar RNA binding specificities and effects on RNA splicing, differential expression of PTB and nPTB can lead to the generation of alternatively spliced mRNAs. RNA binding by PTB and $\mathrm{nPTB}$ is mediated by four RNA recognition motifs (RRMs). We present here the crystal and solution structures of the C-terminal domain of nPTB (nPTB34) which contains RRMs 3 and 4. As expected the structures are similar to each other and to the solution structure of the equivalent fragment from PTB (PTB34). The result confirms that, as found for PTB, RRMs 3 and 4 of nPTB interact with one another to form a stable unit that presents the RNA-binding surfaces of the component RRMs on opposing sides. The major differences between PTB34 and nPTB34 arise from amino acid side chain substitutions on the exposed $\beta$-sheet surfaces and adjoining loops of each RRM, which are likely to modulate interactions with RNA. 
1 Amar Joshi $^{1 * \ddagger}$, Vicent Esteve ${ }^{2 \# \ddagger}$, Adrian N. Buckroyd ${ }^{1 \ddagger}$, Markus Blatter $^{2}$, Frédéric H.-T. Allain ${ }^{2 \dagger}$,

2 Stephen Curry ${ }^{1 \dagger}$

$3 \quad{ }^{1}$ Department of Life Sciences, Imperial College, Exhibition Road, London SW7 2AZ, United

4 Kingdom.

$5 \quad$ Institute of Molecular Biology and Biophysics, ETH Zürich, Switzerland.

6 *Present address: Department of Biochemistry, Henry Wellcome Building, University of

7 Leicester, Lancaster Road, Leicester LE1 9HN, United Kingdom.

8 \#Present address: CIBER-BBN, Dep. Quimica Fisica, Universitat de Valencia, Dr. Moliner, 50

9 46100-Burjassot, Valencia, Spain.

10 Present address: Department of Biochemistry, University of Cambridge, Tennis Court Road,

11 CB2 1QW, United Kingdom.

12 †These authors contributed equally to this work.

\section{Corresponding authors:}

14 Frédéric H.-T. Allain, Institute of Molecular Biology and Biophysics, ETH Zürich, Switzerland.

15 Tel: +41 44633 3940; email: allain@mol.biol.ethz.ch

16 Stephen Curry, Department of Life Sciences, Imperial College, Exhibition Road, London

17 SW7 2AZ, United Kingdom. Tel: +44 207594 7632; email: s.curry@imperial.ac.uk 
Introduction

19 Alternative splicing is the rule rather than the exception in the human genome. Over $95 \%$ of

20 multi-exon genes produce alternatively spliced mRNAs (Nilsen \& Graveley, 2010), yielding

21 many more protein variants or isoforms than specified by the number of genes. The process of

22 alternative splicing is controlled by regulatory sequences within pre-mRNA transcripts that

23 recruit suites of proteins to determine whether selected exons are included or excluded as mRNA

24 is brought to maturity.

25 The polypyrimidine tract binding protein (PTB) is a well-characterized regulator of alternative

26 splicing (García-Blanco, Jamison \& Sharp, 1989; Gil et al., 1991) although it also has significant

27 roles that affect the processing, localization and use of a variety of mRNAs [reviewed by

28 (Sawicka et al., 2008)]. PTB does not recognize a unique RNA sequence; rather it is specific for

29 short motifs (e.g. UCUCU, UCUU) within pyrimidine rich regions of pre-mRNA ( $\underline{\text { Singh, }}$

30 Valcárcel \& Green, 1995; Pérez et al., 1997). In alternative splicing, PTB binding is most

31 commonly associated with exclusion or skipping of the regulated exon, although more recent

32 genome-wide splicing studies have indicated that PTB binding can also promote exon inclusion

33 in a minority of cases ( $\underline{\text { Xue et al., 2009; }}$ Llorian et al., 2010; Witten \& Ule, 2011).

34 Three tissue-specific homologues of PTB have been identified - smPTB, ROD1 and nPTB -

35 all of which have high levels of amino acid sequence identity with the prototypical protein (69-

$3674 \%$ ) and are therefore likely to have very similar structures. smPTB (smooth muscle tissue) has

37 so far only been found in rodents (Gooding, Kemp \& Smith, 2003), whereas the homologue

38 ROD1 is expressed primarily in hematopoietic tissues (Yamamoto et al., 1999). nPTB is found

39 predominantly in brain and testis but also, at low levels, in muscle cells (Markovtsov et al., 2000;

40 Polydorides et al., 2000). 
41 PTB and nPTB bind to the same RNA sequences and have similar effects on alternative splicing

42 events for a number of transcripts (Markovtsov et al., 2000; Pilipenko et al., 2001; $\underline{\text { Spellman, }}$

43 Llorian \& Smith, 2007). However, for some neuronal-specific alternative splicing events the

44 expression of nPTB leads to distinct outcomes. For example, the tyrosine kinase $c$-src has an $n$ -

45 src isoform found only in brain tissue that requires nPTB for the selective inclusion of a

46 neuronal-specific exon (Markovtsov et al., 2000; Sharma, Falick \& Black, 2005; $\underline{\text { Boutz et al., }}$

47 2007).

PTB (and its homologues) contain 4 RNA recognition motif domains (RRMs) separated by long

flexible linkers and arrayed, when not bound to RNA, in a relatively extended, linear

50 conformation (Petoukhov et al., 2006). The solution structures of each RRM have been solved in the absence (Conte et al., 2000; Simpson et al., 2004; Vitali et al., 2006) and presence (Oberstrass

52 et al., 2005) of RNA. While RRMs 1 and 4 adopt a canonical $\beta \alpha \beta \beta \alpha \beta$ topology with two helices

53 packed against a four-strand $\beta$-sheet that forms the primary RNA binding surface, RRMs 2 and 3

54 have a modified architecture. In these domains a C-terminal extension beyond $\beta 4$ loops across the

55 upper edge of the $\beta$-sheet and adds a fifth strand on the far side of the sheet; this expands the size

56 of the RNA binding platform, while the $\beta 4$ - $\beta 5$ loop provides additional points of interaction with

57 RNA (Conte et al., 2000; Simpson et al., 2004; Oberstrass et al., 2005).

58 The $\alpha$-helical regions of RRMs 3 and 4 pack together to form a stable unit in which the RNA-

59 binding $\beta$-sheets are exposed on opposite sides; it has been proposed that this configuration

60 enforces looping of RNA that plays an important role in defining exon structures to be excised

61 from pre-mRNA (Oberstrass et al., 2005; Vitali et al., 2006; Lamichhane et al., 2010). Although

62 early studies had suggested that RRM3 and RRM4 might not interact at physiological salt

63 concentrations ( $\underline{\text { Conte et al., 2000; }}$ Clerte \& Hall, 2006), it is now generally accepted that this

64 pair of RRMs have fixed relative orientations (Lamichhane et al., 2010; Maynard \& Hall, 2010). 
65 Evidence suggests that the RRM1 and RRM2 bind RNA as individual domains although RRM2

66 is also involved in protein-protein interactions with the splicing co-regulator Raver1 (Rideau et

67 al., 2006). Crystallographic analysis has revealed how the helical face of the PTB RRM2

68 provides a binding surface for specific recognition of specific peptide motifs in Raver1 (Joshi et

69 al., 2011).

70 Although PTB and nPTB are likely to have similar structures because of their high sequence

71 identity (75\%) (Fig. 1a), to begin to tease out the structural basis for observed differences in

72 splicing regulation we have determined the structure of a $\mathrm{C}$-terminal fragment of $\mathrm{nPTB}$ that

73 contains RRMs 3 and 4 (nPTB34). We have solved the structures both crystallographically and

74 by NMR. Here we compare those structures with each other and with the solution structure of the 75 equivalent fragment from PTB isoform1 (called here PTB34).

\section{Results and Discussion}

\section{Crystal Structure of nPTB34}

78 nPTB34, a C-terminal nPTB fragment that contains RRMs 3 and 4 (residues 336-531) was over-

79 expressed in E. coli, purified and crystallized by sitting drop vapor diffusion in 20\% PEG 6000 ,

$80 \quad 0.1$ Tris $\mathrm{pH} 8.0$ with $2 \mathrm{mM} \mathrm{ZnCl}$ as an additive (see Materials and Methods). The crystals

81 diffracted X-rays to $1.7 \AA$ and were found to belong to space-group P1. Attempts to phase the

82 diffraction data by molecular replacement using the solution structure of PTB34 (Vitali et al.,

83 2006) were unsuccessful. Instead, phases were obtained by multi-wavelength anomalous

84 dispersion (MAD) using data collected from a crystal of Se-Met-labeled nPTB34. This produced

85 a high-quality electron density map that revealed a total of eight nPTB34 molecules in the

86 asymmetric unit (Fig. 1b). The eight polypeptide chains were built almost in their entirety; due to

87 poor electron density residue 336 at the start of nPTB34 was omitted, as were residues 420-423 
88 (and one or two flanking residues in some chains) at the C-terminal end of the $\beta 4-\beta 5$ loop of

89 RRM3. The final model, which incorporates 788 water molecules, was refined to an $\mathrm{R}_{\text {free }}$ of

$9020.8 \%$ with good stereochemistry (Fig. 1c). Full data collection and refinement statistics are

91 given in Table 1.

92 The structures of the eight copies of nPTB34 within the asymmetric unit of our crystals are very

93 similar to one another (Fig. 1d). Pairwise superpositions of the eight chains give an average root-

94 mean-square deviation (RMSD) for $\mathrm{C}_{\alpha}$ atoms of only $0.17 \AA$. This is not surprising since the unit

95 cell parameters deviate only marginally from a P $2{ }_{1} 2{ }_{1} 2$ space group (see Materials and Methods);

96 what this means is that there are only two positions in the asymmetric unit, exemplified by chains

$97 \mathrm{~A}, \mathrm{~B}, \mathrm{E}$ and $\mathrm{F}$ on the one hand and $\mathrm{C}, \mathrm{D}, \mathrm{G}$ and $\mathrm{H}$ on the other, that have significantly different

98 packing environments.

99 There are a total of $12 \mathrm{Zn}^{2+}$ ions associated with each asymmetric unit which mediate crystal

100 contacts between nPTB34 molecules involving variously His 412, His 491 and His 520. In each

101 case the required tetrahedral coordination appears to be completed by $\mathrm{Cl}^{-}$ions and/or water

102 molecules. These zinc-mediated interactions explain why the cation is required to obtain crystals

103 (see Materials and Methods) but, since only two of the four ligands are provided by amino acids

104 from nPTB34, they are not considered to be physiologically significant.

\section{NMR structure of nPTB34}

106 The solution NMR structure of nPTB34 was obtained from a construct containing residues 325-

107531 and is very similar to the one used for crystallography (see Materials and Methods). Using a

108 total of 3622 nOe derived distance restraints and 55 hydrogen bond restraints identified from

109 slow exchanging amides we derived a fairly good ensemble of nPTB34 conformations in solution 
110 with a heavy atom RMSD of $0.71 \AA$ of the structured region (Fig. 2a). None of the restraints were

111 violated by more than $0.3 \AA$ and $67 \%$ of backbone torsions of the structured residues lay in the

112 most favored region defined by the Ramachandran plot. Due to signal overlaps and broad

113 linewidths the loops ( $\beta 2-\beta 3$-loops, $\beta 4-\beta 5$ loop RRM3) and part of the second beta strand in

114 RRM3 could not be completely assigned. Missing assignments in $\beta 2$ of RRM3 appear to be the

115 reason for the slightly different packing of the first alpha helix $(\alpha 1)$ in RRM3 against the $\beta$-sheet

116 compared to the crystal structure. Since $\alpha 1$ is part of the domain interface this different packing

117 results also in a slight difference in the domain interface compared to the nPTB34 crystal

118 structure and RNA bound and free PTB34 solution structures.

\section{Comparison of Crystal and NMR structures of nPTB34}

120 Overall there is very good correspondence between the structures of nPTB34 determined

121 crystallographically and by NMR. Superposition of the two models using just C atoms gives an

122 RMSD of $3.0 \AA$, and RMSDs of 1.8 and $2.6 \AA$ for RRMs 3 and 4 respectively (Fig. 2b). These

123 values are slightly higher than for the superposition of the crystal structure of nPTB34 onto the

124 solution structures of PTB34 in the absence or presence of RNA, which both give overall RMSDs

125 of $1.3 \AA$ (Fig. 2c). This largely reflects the fact that the solution structure of nPTB34 (unlike

126 PTB34 (Vitali et al., 2006)) was determined without the aid of segmental labeling and thus

127 chemical shift completeness for protons of only $77 \%$ could be achieved. The relative orientation

128 of RRMs 3 and 4 differs slightly between the crystal and solution structures of nPTB34 but this is

129 unlikely to be significant since there is no significant difference in the domain orientations

130 between the crystal structure of nPTB34 and the solution structure of PTB34 (see below);

131 moreover superposition of the crystal structures of the PTB34 fragments from nPTB and hnRNP- 
132 L (

133 is highly conserved between these two proteins (Fig. 2d).

134 Within each RRM of nPTB34 there is good correspondence of secondary structure features

135 between the crystal and solution structures. Those loops in the crystal structure with the highest

136 temperature factors (RRM3 - $\beta 2-\beta 3$ and the C-terminal end of $\beta 4-\beta 5$; RRM4 - $\beta 1-\alpha 2, \beta 2-\beta 3$,

$137 \alpha 2-\beta 4$; and the inter-domain linker) are those exhibiting greatest mobility in the solution

138 structure (Fig. 2a, b). Notably, the $\beta 4-\beta 5$ loop is not disordered in the crystal structure of RRM2

139 but this may simply be because it is involved in more crystal contacts (Joshi et al., 2011).

140 Within the inter-domain linker (residues 433-453), the central portion (residues 439-448) exhibits

141 considerable conformational variation in the solution structure of nPTB34 (Fig. 2a). A similar but

142 slightly smaller region (residues 443-449), which protrudes away from the body of the domain, is

143 variable between the eight chains present in the asymmetric unit of the nPTB34 crystals (Fig. 1d).

144 Again, this likely reflects packing variations since there are effectively only two distinct

145 conformers evident for residues 443-449, which partition into the two distinct packing

146 environments of the pseudo $\mathrm{P} 2{ }_{1} 2_{1} 2_{1}$ space group (see above). The $\mathrm{N}$ - and C-terminal portions of

147 the linker are involved in a number of specific polar and nonpolar interactions that help to

148 stabilize the packing of RRM3 and RRM4 and are described in more detail below.

149 Comparison of nPTB34 with PTB34

150 PTB and $\mathrm{nPTB}$ have $75 \%$ sequence identity overall but this is focused in the RRMs where the

151 sequence identity between equivalent domains ranges from $80 \%$ (RRMs 3 and 4 ) to $88 \%$

152 (RRM2) (Fig. 1a). As expected, the three-dimensional structures of nPTB34 and PTB34 are very

153 similar; differences are largely confined to the local effects of amino acid substitutions. 
154 The crystal structure of nPTB34 reveals that the interaction between RRM3 and RRM4 is

155 stabilized by the exclusion of water molecules, which allows direct contact between a conserved

156 set of hydrophobic residues - Ile 505, Leu 508, Ile 509 and Phe 526 from RRM4 and the apolar

157 portions of Ser 354, Thr 357, Leu 358, Val 361 and Met 400 from RRM3 (Fig. 3a). Ringing this

158 hydrophobic core are direct and water-mediated hydrogen bonds between the RRMs and from the

159 RRMs to the linker polypeptide. Within the RRM3-RRM4 interface there are only five amino

160 acids changes between PTB34 and nPTB34 and these are generally of a conservative character;

161 the apolar residues Ile 356, Met 400 and Val 505 in PTB34 are replaced by Thr 357, Leu 399 and

162 Ile 505 respectively in nPTB34, while His 397 and His 400 in PTB34 are altered to Gln 398 and

163 Tyr 401 (Table 3). The water-mediated hydrogen bond interaction from Tyr 401 of RRM3 to the

164 side chain of Asp 510 in RRM4 of nPTB34 is therefore lost in PTB34 but neither this nor the

165 other amino acid changes appear sufficient to significantly alter the interaction between the two

166 domains. A similar degree of conservation of the apolar residues at the hydrophobic interface

167 between RRM3 and RRM4 is also seen in hnRNP-L (Zhang et al., 2013) (Table 3). The relative

168 orientation of these domains is therefore a conserved feature of PTB, nPTB and hnRNP-L. The

169 incorporation of a pair of His residues within the inter-domain interface of PTB34 (H397, H400)

170 may affect the stability of the module at low $\mathrm{pH}$ (Nordlund et al., 2003), but we are not aware of

171 any circumstances where $\mathrm{pH}$ regulation of the conformation would be applied.

172 Although the interaction of RRM4 with the helical face of RRM3 within PTB34 and nPTB34 is

173 much more extensive than the interaction of PTB-binding peptides in Raver1 with the equivalent

174 face of RRM2 (Joshi et al., 2011), there is one interesting parallel. Superposition of the RRM2-

175 raver1 co-crystal structure onto RRM3 of nPTB34 reveals that the Raver1 peptide occupies

176 essentially the same position as helix $\alpha 2$ of RRM4 (Fig. 3b,c). Although the Raver1 peptide is

177 non-helical — in fact it has a pinched but largely extended conformation — it inserts a pair of 
178 Leu residues (Leu 500 and Leu 501 in PDB entry 3zzy) into a hydrophobic pocket formed

179 between the helices $\alpha 1$ and $\alpha 2$ of RRM2 that effectively mimics the positioning of Ile 505 and

180 Ile 509 from RRM4 into the equivalent pocket on RRM3 within nPTB34.

181 Although the RRMs are the most highly conserved features between PTB and nPTB, there are

182 some notable differences on their RNA binding surfaces. These differences appear to be more

183 concentrated on RRM3. While there are a total of five amino acid substitutions in nPTB RRM3

184 of residues identified to contact RNA in the solution structure of the complex of PTB34 with a

185 CUCUCU oligonucleotide (Oberstrass et al., 2005), there are only two such amino acid changes

186 in RRM4 (Fig. 4).

187 The replacement of Phe 371 in the $\beta 2-\beta 3$ loop of RRM3 in PTB34 by Tyr 372 in nPTB34 is

188 conservative and likely to preserve the stacking interaction with the cytosine base at position 5 in

189 CUCUCU RNA oligomer. However, the substitution of Asn 376 (to Ser 377) at the start of the $\beta 3$

190 strand and a triplet of replacements in the $\beta 4$ - $\beta 5$ loop (Asn 413, Gln 421 and Glu 422 in PTB34

191 to Thr 414, Leu 422 and Asp 423 respectively in nPTB34) may modulate RNA binding (Fig. 4a).

192 Likewise the $\beta 2-\beta 3$ loop of RRM4 in PTB34 is altered in nPTB34 by deletion of Lys 489 and

193 replacement of Arg 491 by His, a pair of changes that may also alter RNA interactions (Fig. 4b).

194 The idea that these structural changes affect RNA affinity is supported by mutagenesis

195 experiments which showed that residues in the $\beta 2-\beta 3$ loops of RRMs 3 and 4 and in the $\beta 4-\beta 5$

196 loop of RRMs are important for RNA binding (Conte et al., 2000; Yuan et al., 2002). The

197 observed amino acid changes are likely to contribute to the differences observed in the

198 interactions of PTB and nPTB with larger RNA targets (Pilipenko et al., 2001). However, it

199 should not be forgotten that sequence differences between PTB and nPTB are focused in regions

200 outside the RRM domains and these may well also be important for differences in splicing 
activity (Markovtsov et al., 2000; Robinson \& Smith, 2005), perhaps by recruiting different

202 protein cofactors.

\section{Materials and Methods}

204 Plasmid construction and protein expression and purification of nPTB34 for crystal structure determination

206

207

208

209

210

211

212

213

214

215

216

217

218

219

220

221

222

223

The cDNA coding for residues 336-531 of nPTB were amplified by PCR and ligated into the $E$. coli expression vector pETM-11 (Zou et al., 2003), which adds an N-terminal hexahistidine tag that could be removed using the Tobacco Etch Virus N1a protease. The fragment was cloned to position the first residue (G336) as the Gly in the TEV protease recognition site (ENLYFQ/G); protease processing therefore removed all vector-derived amino acids.

Expression of nPTB34 was induced in E. coli BL21 (DE3) cells at $37^{\circ} \mathrm{C}$ for four hours by addition of $1 \mathrm{mM}$ isopropyl $\beta$-D-1-thiogalactopyranoside (IPTG). Cells were re-suspended in $0.1 \mathrm{M} \mathrm{NaCl}, 25 \mathrm{mM}$ Tris $\mathrm{HCl} \mathrm{pH} 7.5,0.1 \%$ Triton X-100, $0.5 \mathrm{mM}$ phenylmethylsulfonyl fluoride (PMSF) and $1 \mathrm{mg} / \mathrm{mL}$ lysozyme and lysed by sonication. Cell debris and nucleic acid were removed by centrifugation at $25,000 \mathrm{~g}$ for 40 minutes at $4^{\circ} \mathrm{C}$ in the presence of $1 \mathrm{mg} / \mathrm{mL}$ protamine sulfate. nPTB34 was purified from the supernatant by gravity flow through TALON Metal Affinity Resin (Clontech) with stepped elution. The hexahistadine tag was cleaved by addition of 1mg Tobacco Etch Virus N1a protease for $20 \mathrm{mg}$ of nPTB34 and dialysed overnight into $50 \mathrm{mM}$ Tris $\mathrm{pH} 7.8$, containing $0.1 \mathrm{M} \mathrm{NaCl}, 10 \%$ glycerol, $3 \mathrm{mM}$ ßmercaptoethanol and 0.5 $\mu \mathrm{M}$ Ethylenediamine tetraacetic acid (EDTA). Cleaved nPTB34 was purified away from the tag, uncleaved nPTB34 and N1a protease by a second application to TALON resin. Proteins were further purified by size exclusion chromatography on a Superdex 75 (GE Healthcare) using an ÄKTA FPLC system in $25 \mathrm{mM}$ Tris pH 7.8, $0.1 \mathrm{M} \mathrm{NaCl}$ and $0.5 \mathrm{mM}$ DTT. Peak fractions were 
concentrated and stored at $-80^{\circ} \mathrm{C}$. Expression of Se-Methionine (Se-Met) labeled nPTB34 was

225 performed as above with the following differences: proteins were expressed in E. coli B834

226 (DE3) cells in minimal media supplemented with Se-Met (Molecular Dimensions). Purification

227 proceeded as for unlabeled protein with the addition of $5 \mathrm{mM} \beta$-mercaptoethanol to all buffers.

228 Incorporation of Se-Met was determined to be $100 \%$ by MALDI-TOF mass spectrometry (data 229 not shown).

\section{Crystal structure determination of nPTB34}

231 Purified recombinant $\mathrm{nPTB} 34$ was concentrated to $\approx 5 \mathrm{mg} / \mathrm{mL}$ in $100 \mathrm{mM} \mathrm{NaCl}, 20 \mathrm{mM}$ Tris ( $\mathrm{pH}$

232 7.8) and $0.5 \mathrm{mM}$ DTT. nPTB34 was crystallised by sitting drop vapor diffusion with a reservoir

233 solution containing 0.1 M Tris ( $\mathrm{pH} 8.0$ ), $2 \mathrm{mM} \mathrm{ZnCl}_{2}$ and 20\% polyethylene glycol (PEG) 6000.

234 Crystals appeared at first as stacked plates, but single crystals were obtained after micro-seeding 235 into equilibrated drops of nPTB34 in $0.1 \mathrm{M}$ Tris ( $\mathrm{pH} 8.0$ ), 0.2 $\mathrm{mM} \mathrm{ZnCl}_{2}$ and 12\% PEG 6000. No

236 crystals were obtained in the absence of $\mathrm{ZnCl}_{2}$ - the structure revealed that this is because two 237 crystal-packing interactions are mediated by zinc ions (see results). Crystals were harvested and 238 briefly incubated in mother liquor supplemented with $20 \%$ glycerol before flash cooling in liquid $239 \mathrm{~N}_{2}$.

240 X-ray diffraction data were collected from a single crystal $(60 \mu \mathrm{m} \times 40 \mu \mathrm{m} \times 20 \mu \mathrm{m})$ at beamline

241 I02 at the Diamond Light Source (Didcot, UK). Diffraction data were initially indexed in

242 iMosflm and scaled in space group P2 $2_{1} 2$ using SCALA (Collaborative Computer Project No. 4,

243 1994)( RRID:nif-0000-30238). Closer inspection of the data in POINTLESS suggested the space 244 group was more likely to be $\mathrm{P} 2{ }_{1}$. At this stage molecular replacement was attempted in PHASER

245 (McCoy et al., 2007) using the solution structure of nPTB34 as a search model to locate the four 
246 molecules in the asymmetric unit expected from the Matthews co-efficient of $2.08 \mathrm{Da} \AA^{-3}$ but

247 failed to find any acceptable solutions.

248 We therefore switched to phasing by multi-wavelength anomalous dispersion (MAD) using 249 isomorphous crystals grown from Se-Met labeled nPTB34 (see above). Diffraction data were 250 collected at three wavelengths at beamline I03 at the Diamond Light Source and processed in 251 space-group P2 $2_{1}$ Heavy atom site were identified using SHELXD within autoSHARP (Bricogne 252 et al., 2003; Vonrhein et al., 2007; Sheldrick, 2008) and following density modification the figure 253 of merit was 0.802 . Initial model building was performed automatically in BUCCANEER 254 (Cowtan, 2006) where two nPTB34 molecules were built in the asymmetric unit. We used this 255 initial model in Phaser 2.1 to find the remaining pair of molecules in the asymmetric unit.

256 However, maps calculated using the 'completed' model remained noisy in the region 257 corresponding to this second pair of polypeptide chains and refinement stalled at an $R_{\text {free }}$ value of 258 over $37 \%$. At this stage the data were re-indexed in P1 and a molecular replacement search 259 performed using the tetrameric arrangement of crystallographic models generated as described 260 above was used to locate all eight 8 copies of nPTB34 in the larger asymmetric unit. This model 261 was adjusted manually in COOT (Emsley \& Cowtan, 2004) and refined without any further 262 difficulty. Initially, strict NCS restraints were employed during refinement in CNS v1.2 (Brunger 263 et al., 1998). TLS refinement and latter cycles of refinement were performed in Phenix without

264 NCS restraints (Adams et al., 2010), yielding an $\mathrm{R}_{\text {free }}$ of $27.3 \%$. At this stage the structure was 265 deposited in the Protein Data Bank (PDB; RRID:nif-0000-00135) under ID 4cko, the manuscript 266 published as a preprint ( $\underline{\text { Joshi et al., 2014) }}$ and submitted for peer review. One reviewer, noting

267 the relatively high value of $\mathrm{R}_{\text {free}}$, raised the possibility that the crystals might be twinned,

268 something we had overlooked while resolving the problems due to the initial mis-assignment of 269 the space-group. Analysis using the xtriage function in PHENIX revealed the presence of 
twinning operators around each of the three principal axes. Re-refinement in REFMAC (Winn.

271 Murshudov \& Papiz, 2003) (with the presence of 4 twin domains with the following twin

272 operators and fractions: h, k, $1-0.153 ;-h,-k, 1-0.245 ; \mathrm{h},-\mathrm{k},-1-0.176 ;-\mathrm{h}, \mathrm{k},-1-0.425)$

273 significantly reduced the values of $\mathrm{R}_{\text {work }}$ and $\mathrm{R}_{\text {free }}$ (to $17.0 \%$ and $20.8 \%$ respectively) but did not

274 appreciably improve the electron density map, which was already of high quality.

275 Stereochemistry and clashes were assessed using MolProbity (Adams et al., 2010). The final

276 refined coordinates and structure factors have been deposited with the PDB (ID 4cq1).

277 Plasmid construction, expression and purification of nPTB34 for solution structure.

278 A 207-residue C-terminal fragment (residues 325-531) of human nPTB (Swissprot Q9UKA9), 279 which contain the third and fourth RRMs, was sub-cloned into a pET-28a(+) vector, using the 280 restriction sites Nde I and Not I. The sequence contained an N-terminal 6xHis-tag. The proteins 281 were over-expressed in BL21(+) E.coli cultures grown in M9 minimal medium containing 50

$282 \mathrm{~g} / \mathrm{mL}$ Kanamycin. The expression was induced by IPTG at an OD of 0.4. For ${ }^{15} \mathrm{~N}$-labelling ${ }^{15} \mathrm{~N}-$

$283 \mathrm{NH}_{4} \mathrm{Cl}$ was added as the only nitrogen source and for ${ }^{13} \mathrm{C}$-labelling normal glucose was replaced

284 by ${ }^{13} \mathrm{C}$ labeled glucose. The cell pellets were harvested by centrifugation and resuspended in lysis

285 buffer containing $300 \mathrm{mM} \mathrm{NaCl}, 50 \mathrm{mM} \mathrm{NaH}_{2} \mathrm{PO}_{4}$ and $1 \mathrm{mM}$ imidazole at $\mathrm{pH} 8$ and lysed by 3

286 passages through a cell-cracker (EmulsiFlex-C5 High Pressure Homogenizer from Avestin,

287 Canada). The protein was purified by two successive Ni-NTA columns ( $1 \mathrm{ml} \mathrm{Ni-NTA} \mathrm{resin} \mathrm{per}$

288 liter of culture). Elution occurred between $40 \mathrm{mM}$ and $200 \mathrm{mM}$ imidazole. After the second

289 column the protein was dialyzed against the NMR buffer containing $20 \mathrm{mM} \mathrm{NaCl}$ and $10 \mathrm{mM}$

$290 \mathrm{NaH}_{2} \mathrm{PO}_{4}$ at $\mathrm{pH} 5.8$ and concentrated by centricon ultra centrifugation to 1 to $2 \mathrm{mM}$. The purity of

291 the protein sample was tested by SDS gels. The final yield per liter of culture was estimated to be

292 around $20 \mathrm{mg}$ protein. Mass spectroscopy was used to characterize the proteins. 


\section{NMR spectroscopy}

294 Shigemi NMR tubes with $250 \mathrm{~L}$ sample solution $\left(10 \%\right.$ or $\left.100 \% \mathrm{D}_{2} \mathrm{O}\right)$ were used. NMR spectra 295 were acquired at $303 \mathrm{~K}$ on a Bruker DRX-600 and a Avance-900 spectrometer. NMR data were 296 processed using Topspin 3.0 (Bruker) and analyzed using Sparky (Goddard \& Kneller, 2007).

297 For the sequence specific backbone assignment HNCA, HN(CO)CA, CBCA(CO)NH, HNCACB, $298{ }^{15} \mathrm{~N}$ HSQC,${ }^{13} \mathrm{C}$ HSQC as well as ${ }^{15} \mathrm{~N}$ TROSY spectra were recorded. The aliphatic side chains 299 were assigned based on a ${ }^{15} \mathrm{~N}$ NOESY and a ${ }^{13} \mathrm{C}$ NOESY with the help of a ${ }^{15} \mathrm{~N}$ TOCSY and a

$300 \mathrm{HCCH}-\mathrm{TOCSY}$. The assignment of side chain amides was achieved by analyzing the ${ }^{15} \mathrm{~N}$ NOESY 301 and the ${ }^{15} \mathrm{~N}$ HSQC, while for the assignment of the aromatic side chains a 2D TOCSY and a 2D

302 NOESY in $\mathrm{D}_{2} \mathrm{O}$ were recorded. Distance restraints used in structure calculations were extracted 303 from ${ }^{15} \mathrm{~N}-\mathrm{NOESY}-\mathrm{HSQC}$ and ${ }^{13} \mathrm{C}-\mathrm{NOESY}-\mathrm{HSQC}$ in $\mathrm{H}_{2} \mathrm{O}$ and 2D NOESY in $\mathrm{D}_{2} \mathrm{O}$. Hydrogen-

304 bonded $\mathrm{NH}$ groups were identified by the presence of amide resonances in ${ }^{15} \mathrm{~N} \mathrm{HSQC}$ spectra, 305 which were recorded immediately after lyophilizing and dissolving the sample in $\mathrm{D}_{2} \mathrm{O}$.

\section{NMR structure calculation}

307 Initial peak picking and nOe assignments were performed using the ATNOSCANDID package 308 (Herrmann, Guntert \& Wuthrich, 2002b; Herrmann, Guntert \& Wuthrich, 2002a). Peak lists of 309 the final seventh cycle were used as an input for the program CYANA 3.0 (Guntert, 2004). The 310 "noeassign" protocol of CYANA was used to reassign and calibrate the nOe signals of the given 311 peak lists. These lists were manually reviewed and the distance restraint list were further used by

312 CYANA to calculated 250 structures by a simulated annealing protocol (20,000 MD steps). Based

313 on the target function the 50 best structures were selected for refinement using a simulated 314 annealing protocol with the AMBER 9 suite (Pearlman et al., 1995) and against the ff99 force 
315 field (Lindorff-Larsen et al., 2010) using implicit water (Bashford \& Case, 2000). The 20 final

316 conformers were selected using a combined AMBER energy and violation energy as selection

317 criteria. The coordinates have been deposited in the Protein Data Bank (ID 2mju); NMR chemical

318 shifts and distance constraints used in the structure calculation have been deposited under ID

31919737 to the Biological Magnetic Resonance Data Bank (BMRB; RRID:nif-0000-21058).

\section{Acknowledgements}

321 We thank staff at the Diamond Light Source (Didcot, UK) for assistance with X-ray data

322 collection. We are grateful to Reviewer 3 for drawing our attention to the possibility that the

323 diffraction data might be twinned.

\section{References}

325 Adams PD, Afonine PV, Bunkoczi G, Chen VB, Davis IW, Echols N, Headd JJ, Hung LW, 326 Kapral GJ, Grosse-Kunstleve RW, McCoy AJ, Moriarty NW, Oeffner R, Read RJ, Richardson 327 DC, Richardson JS, Terwilliger TC, and Zwart PH. 2010. PHENIX: a comprehensive Python328 based system for macromolecular structure solution. Acta Cryst D 66:213-221.

329 Bashford D, and Case DA. 2000. Generalized born models of macromolecular solvation effects. $330 \quad$ Annu Rev Phys Chem 51:129-152.

331 Boutz PL, Stoilov P, Li Q, Lin C-H, Chawla G, Ostrow K, Shiue L, Ares M, and Black DL. 2007. 332 A post-transcriptional regulatory switch in polypyrimidine tract-binding proteins reprograms 333 alternative splicing in developing neurons. Genes Dev 21:1636-1652. 
334 Bricogne G, Vonrhein C, Flensburg C, Schiltz M, and Paciorek W. 2003. Generation, 335 representation and flow of phase information in structure determination: recent developments 336 in and around SHARP 2.0. Acta Cryst D 59:2023-2030.

337 Brunger AT, Adams PD, Clore GM, DeLano WL, Gros P, Grosse-Kunstleve RW, Jiang JS, 338 Kuszewski J, Nilges M, Pannu NS, Read RJ, Rice LM, Simonson T, and Warren GL. 1998. 339 Crystallography \& NMR system: A new software suite for macromolecular structure 340 determination. Acta Cryst D 54:905-921.

341 Clerte C, and Hall KB. 2006. Characterization of multimeric complexes formed by the human 342 PTB1 protein on RNA. RNA (New York, NY) 12:457-475.

343 Collaborative Computer Project No. 4. 1994. The CCP4 suite: programs for protein 344 crystallography. Acta Cryst D 50:760-763.

345 Conte MR, Grüne T, Ghuman J, Kelly G, Ladas A, Matthews S, and Curry S. 2000. Structure of 346 tandem RNA recognition motifs from polypyrimidine tract binding protein reveals novel 347 features of the RRM fold. EMBO J 19:3132-3141.

348 Cowtan K. 2006. The Buccaneer software for automated model building. 1. Tracing protein 349 chains. Acta Cryst D 62:1002-1011.

350 Emsley P, and Cowtan K. 2004. Coot: model-building tools for molecular graphics. Acta Cryst D $351 \quad 60: 2126-2132$.

352 García-Blanco MA, Jamison SF, and Sharp PA. 1989. Identification and purification of a 62,000353 dalton protein that binds specifically to the polypyrimidine tract of introns. Genes Dev $354 \quad 3: 1874-1886$.

355 Gil A, Sharp PA, Jamison SF, and Garcia-Blanco MA. 1991. Characterization of cDNAs 356 encoding the polypyrimidine tract-binding protein. Genes Dev 5:1224-1236.

357 Goddard TD, and Kneller DG. 2007. SPARKY 3.114. San Francisco: University of California. 
Gooding C, Kemp P, and Smith CWJ. 2003. A novel polypyrimidine tract-binding protein paralog expressed in smooth muscle cells. J Biol Chem 278:15201-15207.

360 Guntert P. 2004. Automated NMR structure calculation with CYANA. Methods Mol Biol 278:353-378.

362 Herrmann T, Guntert P, and Wuthrich K. 2002a. Protein NMR structure determination with 363 automated NOE assignment using the new software CANDID and the torsion angle dynamics 364 algorithm DYANA. J Mol Biol 319:209-227.

365 Herrmann T, Guntert P, and Wuthrich K. 2002b. Protein NMR structure determination with 366 automated NOE-identification in the NOESY spectra using the new software ATNOS. $J$ Biomol NMR 24:171-189.

Joshi A, Coelho MB, Kotik-Kogan O, Simpson PJ, Matthews SJ, Smith CW, and Curry S. 2011.

369 Crystallographic analysis of polypyrimidine tract-binding protein-raver1 interactions involved in regulation of alternative splicing. Structure 19:1816-1825.

Joshi A, Esteve V, Buckroyd AN, Blatter M, Allain FH, and Curry S. 2014. Solution and crystal structures of a C-terminal fragment of the neuronal isoform of the polypyrimidine tract binding protein (nPTB). PeerJ Preprints 2:e211.

374 Lamichhane R, Daubner GM, Thomas-Crusells J, Auweter SD, Manatschal C, Austin KS, 375 Valniuk O, Allain FH-T, and Rueda D. 2010. RNA looping by PTB: Evidence using FRET 376 and NMR spectroscopy for a role in splicing repression. Proc Natl Acad Sci USA 107:41054110.

Lindorff-Larsen K, Piana S, Palmo K, Maragakis P, Klepeis JL, Dror RO, and Shaw DE. 2010. 379 Improved side-chain torsion potentials for the Amber ff99SB protein force field. Proteins 78:1950-1958.

381 Llorian M, Schwartz S, Clark TA, Hollander D, Tan L-Y, Spellman R, Gordon A, Schweitzer AC, 382 De La Grange P, Ast G, and Smith CWJ. 2010. Position-dependent alternative splicing 
activity revealed by global profiling of alternative splicing events regulated by PTB. Nat Struct Mol Biol 17:1114-1123.

385 Markovtsov V, Nikolic JM, Goldman JA, Turck CW, Chou MY, and Black DL. 2000.

386 Cooperative assembly of an hnRNP complex induced by a tissue-specific homolog of 387 polypyrimidine tract binding protein. Mol Cell Biol 20:7463-7479.

388 Maynard CM, and Hall KB. 2010. Interactions between PTB RRMs induce slow motions and 389 increase RNA binding affinity. J Mol Biol 397:260-277.

390 McCoy AJ, Grosse-Kunstleve RW, Adams PD, Winn MD, Storoni LC, and Read RJ. 2007. 391 Phaser crystallographic software. J Appl Crystallogr 40:658-674.

392 Nilsen TW, and Graveley BR. 2010. Expansion of the eukaryotic proteome by alternative 393 splicing. Nature 463:457-463.

394 Nordlund HR, Hytönen VP, Laitinen OH, Uotila STH, Niskanen EA, Savolainen J, Porkka E, and 395 Kulomaa MS. 2003. Introduction of histidine residues into avidin subunit interfaces allows pH-dependent regulation of quaternary structure and biotin binding. FEBS Letters 555:449454.

Oberstrass FC, Auweter SD, Erat M, Hargous Y, Henning A, Wenter P, Reymond L, AmirAhmady B, Pitsch S, Black DL, and Allain FH-T. 2005. Structure of PTB bound to RNA: specific binding and implications for splicing regulation. Science 309:2054-2057.

Pearlman DA, Case DA, Caldwell JW, Ross WS, Cheatham Iii TE, DeBolt S, Ferguson D, Seibel G, and Kollman P. 1995. AMBER, a package of computer programs for applying molecular mechanics, normal mode analysis, molecular dynamics and free energy calculations to simulate the structural and energetic properties of molecules. Comput Phys Commun 91:1-41.

Pérez I, Lin CH, McAfee JG, and Patton JG. 1997. Mutation of PTB binding sites causes misregulation of alternative 3 ' splice site selection in vivo. RNA 3:764-778. 
407 Petoukhov MV, Monie TP, Allain FH, Matthews S, Curry S, and Svergun DI. 2006.

408 Conformation of polypyrimidine tract binding protein in solution. Structure 14:1021-1027.

409 Pilipenko EV, Viktorova EG, Guest ST, Agol VI, and Roos RP. 2001. Cell-specific proteins

410 regulate viral RNA translation and virus-induced disease. EMBO J 20:6899-6908.

411 Polydorides AD, Okano HJ, Yang YY, Stefani G, and Darnell RB. 2000. A brain-enriched 412 polypyrimidine tract-binding protein antagonizes the ability of Nova to regulate neuron$413 \quad$ specific alternative splicing. Proc Natl Acad Sci USA 97:6350-6355.

414 Rideau AP, Gooding C, Simpson PJ, Monie TP, Lorenz M, Hüttelmaier S, Singer RH, Matthews 415 S, Curry S, and Smith CWJ. 2006. A peptide motif in Raver1 mediates splicing repression by 416 interaction with the PTB RRM2 domain. Nat Struct Mol Biol 13:839-848.

417 Robinson F, and Smith CW. 2005. A splicing repressor domain in polypyrimidine tract binding 418 protein. J Biol Chem.

419 Sawicka K, Bushell M, Spriggs KA, and Willis AE. 2008. Polypyrimidine-tract-binding protein: a $420 \quad$ multifunctional RNA-binding protein. Biochem Soc Trans 36:641-647.

421 Sharma S, Falick AM, and Black DL. 2005. Polypyrimidine tract binding protein blocks the 5' 422 splice site-dependent assembly of U2AF and the prespliceosomal E complex. Mol Cell $423 \quad 19: 485-496$.

424 Sheldrick GM. 2008. A short history of SHELX. Acta Cryst A 64:112-122.

425 Simpson PJ, Monie TP, Szendröi A, Davydova N, Tyzack JK, Conte MR, Read CM, Cary PD, 426 Svergun DI, Konarev PV, Curry S, and Matthews S. 2004. Structure and RNA interactions of 427 the N-terminal RRM domains of PTB. Structure 12:1631-1643.

428 Singh R, Valcárcel J, and Green MR. 1995. Distinct binding specificities and functions of higher 429 eukaryotic polypyrimidine tract-binding proteins. Science 268:1173-1176.

430 Spellman R, Llorian M, and Smith CWJ. 2007. Crossregulation and functional redundancy 431 between the splicing regulator PTB and its paralogs nPTB and ROD1. Mol Cell 27:420-434. 
432 Vitali F, Henning A, Oberstrass FC, Hargous Y, Auweter SD, Erat M, and Allain FH-T. 2006.

433 Structure of the two most C-terminal RNA recognition motifs of PTB using segmental 434 isotope labeling. EMBO J 25:150-162.

435 Vonrhein C, Blanc E, Roversi P, and Bricogne G. 2007. Automated structure solution with 436 autoSHARP. Methods Mol Biol 364:215-230.

437 Winn MD, Murshudov GN, and Papiz MZ. 2003. Macromolecular TLS refinement in REFMAC 438 at moderate resolutions. Methods in enzymology 374:300-321.

439 Witten JT, and Ule J. 2011. Understanding splicing regulation through RNA splicing maps. $440 \quad$ Trends Genet 27:89-97.

441 Xue Y, Zhou Y, Wu T, Zhu T, Ji X, Kwon Y-S, Zhang C, Yeo G, Black DL, Sun H, Fu X-D, and 442 Zhang Y. 2009. Genome-wide analysis of PTB-RNA interactions reveals a strategy used by 443 the general splicing repressor to modulate exon inclusion or skipping. Mol Cell 36:996-1006.

444 Yamamoto H, Tsukahara K, Kanaoka Y, Jinno S, and Okayama H. 1999. Isolation of a 445 mammalian homologue of a fission yeast differentiation regulator. Mol Cell Biol 19:38294463841.

447 Yuan X, Davydova N, Conte MR, Curry S, and Matthews S. 2002. Chemical shift mapping of 448 RNA interactions with the polypyrimidine tract binding protein. Nucleic Acids Res 30:456449462.

450 Zhang W, Zeng F, Liu Y, Zhao Y, Lv H, Niu L, Teng M, and Li X. 2013. Crystal structures and 451 RNA-binding properties of the RNA recognition motifs of heterogeneous nuclear 452 ribonucleoprotein L: insights into its roles in alternative splicing regulation. J Biol Chem $453 \quad 288: 22636-22649$.

454 Zou P, Gautel M, Geerlof A, Wilmanns M, Koch MHJ, and Svergun DI. 2003. Solution scattering 455 suggests cross-linking function of telethonin in the complex with titin. J Biol Chem 278:26364562644. 


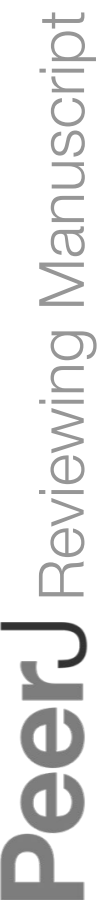

PeerJ reviewing PDF | (v2014:01:1378:1:0:NEW 14 Feb 2014) 


\section{Table 1 (on next page)}

Crystal structure - data collection and refinement statistics 
Table 1: Crystal structure - data collection and refinement statistics

\begin{tabular}{|c|c|c|c|c|}
\hline $\begin{array}{l}\text { DATA } \\
\text { COLLECTION }\end{array}$ & & & & \\
\hline Space-group & $\mathrm{P} 1$ & \multicolumn{3}{|c|}{$\mathrm{P} 2_{1}$} \\
\hline a, b, c $(\AA)$ & $61.00,65.81,99.58$ & \multicolumn{3}{|c|}{$65.81,60.95,99.55$} \\
\hline$\alpha, \beta, \gamma\left(^{\circ}\right)$ & $89.99,90.00,89.99$ & \multicolumn{3}{|c|}{$90.00,90.01,90.00$} \\
\hline & & Peak & Inflection & Remote \\
\hline Wavelength $(\AA ̊)$ & 0.9795 & 0.9808 & 0.9813 & 0.9790 \\
\hline Resolution range (̊̊) & $\begin{array}{l}44.74-1.69 \\
(1.72-1.69)\end{array}$ & $\begin{array}{l}55.10-2.30 \\
(2.42-2.30)\end{array}$ & $\begin{array}{l}55.14-2.30 \\
(2.42-2.30)\end{array}$ & $\begin{array}{l}55.27-2.50 \\
(2.64-2.50)\end{array}$ \\
\hline $\begin{array}{l}\text { No. of independent } \\
\text { reflections }\end{array}$ & $153732(7340)$ & - & - & - \\
\hline Multiplicity $^{1}$ & $1.9(1.9)$ & $1.7(1.6)$ & $1.7(1.7)$ & $1.7(1.7)$ \\
\hline Completeness (\%) & $89.0(85.6)$ & $89.9(84.8)$ & $95.9(96.1)$ & $95.7(93.5)$ \\
\hline$I / \sigma_{I}$ & $5.0(1.1)$ & $10.0(4.3)$ & $8.7(3.0)$ & $8.1(2.4)$ \\
\hline $\mathbf{R}_{\text {merge }}(\%)^{2}$ & $9.6(70.7)$ & $6.7(21.2)$ & $8.6(37.9)$ & $9.4(47.8)$ \\
\hline $\begin{array}{l}\text { MODEL } \\
\text { REFINEMENT }\end{array}$ & $40.81-1.69 \AA ̊$ & & & \\
\hline $\begin{array}{l}\text { No. of Non-hydrogen } \\
\text { atoms/waters }\end{array}$ & 12397 & & & \\
\hline $\mathbf{R}_{\text {work }}(\%)^{3}$ & 17.00 & & & \\
\hline $\mathbf{R}_{\text {free }}(\%)^{4}$ & 20.76 & & & \\
\hline RMS bonds $(\AA)^{5}$ & 0.006 & & & \\
\hline RMS bond angles $\left({ }^{\circ}\right)$ & 0.937 & & & \\
\hline $\begin{array}{l}\text { Ramachandran plot } \\
\text { (\% favoured/allowed) }\end{array}$ & $97.4 / 1.9$ & & & \\
\hline PDB Identifier & $4 \mathrm{cq} 1$ & & & \\
\hline
\end{tabular}

${ }^{1}$ Values for highest resolution shell given in parentheses

${ }^{2} \mathrm{R}_{\text {merge }}=100 \quad \sum_{\mathrm{hkl}}\left|\mathrm{I}_{\mathrm{j}}(\mathrm{hkl})-\left\langle\mathrm{I}_{\mathrm{j}}(\mathrm{hkl})\right\rangle\right| / \sum_{\mathrm{hkl}} \Sigma_{\mathrm{j}} \mathrm{I}(\mathrm{hkl})$, where $\mathrm{I}_{\mathrm{j}}(\mathrm{hkl})$ and $\left\langle\mathrm{I}_{\mathrm{j}}(\mathrm{hkl})\right\rangle$ are the intensity of measurement $\mathrm{j}$ and the mean intensity for the reflection with indices hkl, respectively.

${ }^{3} \mathrm{R}_{\text {work }}=100 \quad \sum_{\mathrm{hkl}}|| \mathrm{F}_{\text {obs }}|-| \mathrm{F}_{\text {calc }}|| / \sum_{\mathrm{hkl}}\left|\mathrm{F}_{\text {obs }}\right|$.

${ }^{4} R_{\text {free }}$ is the $R_{\text {work }}$ calculated using a randomly selected $5 \%$ sample of reflection data that were omitted from the refinement.

${ }^{5} \mathrm{RMSD}$, root-mean-squared deviations. 


\section{Table 2 (on next page)}

Statistics for the solution structure of nPTB34 
1 Table 2: Statistics for the solution structure of nPTB34

$\begin{array}{ll}\text { Number of Distance Restraints } & 3677 \\ \text { intraresidual } & 657 \\ \text { sequential }(|\mathrm{i}-\mathrm{j}|=1) & 941 \\ \text { medium range }(1<|\mathrm{i}-\mathrm{j}|<5) & 684 \\ \text { long range }(|\mathrm{i}-\mathrm{j}|>=5) & 134 \\ \text { hydrogen bonds }^{1} & 55\end{array}$

Energy Statistics ${ }^{2}$

Average distance constraint violations $(\AA)$
$0.1-0.2 \AA$
$0.2-0.3 \AA$
$52.4+/-4.2$
$>0.3 \mathrm{~A}$
$0.4+/-0.6$
Maximal (̊)
$0.0+/-0.0$
$0.20+/-0.03$

Mean AMBER distance violation Energy (kcal $100.9+/-2.5$

$\mathrm{mol}^{-1}$ )

Mean AMBER Energy ( $\left.\mathrm{kcal} \mathrm{mol}^{-1}\right)$

$-6170.0+/-15.5$

Mean Deviation from ideal covalent geometry

Bond Length (A)

$0.0042+/-$

0.0000

Bond Angle (degrees)

$1.796+/-0.007$

Ramachandran plot Statistics $2,3,4$

Most favoured regions (\%)

$67.1+/-2.0$

Additionally allowed regions (\%)

$29.2+/-1.9$

Generously allowed regions (\%)

$3.5+/-0.8$

Disallowed regions (\%)

$0.1+/-0.2$

RMS Deviations from mean structure

Statistics 2,3
Backbone atoms $(\AA)$
$0.37+/-0.05$
Heavy atoms $(\AA)$
$0.71+/-0.05$

PDB identifier

$2 \mathrm{mju}$

2

$3 \quad{ }^{1}$ Hydrogen bond constraints were identified from slow exchanging amide

4 protons in $\mathrm{D}_{2} \mathrm{O}$.

$5 \quad{ }^{2}$ Statistics computed for the deposited bundle of 20 violation energy best

6 structures selected out of 30 energy best structures refined in Amber

7 (Pearlman et al., 1995).

$8{ }^{3}$ Based on structured residue range as defined by cyana command

9 overlay: 9-47, 52-86, 91-113, 126-163, 168-207.

$10{ }^{4}$ Ramachandran plot, as defined by the Procheck (Laskowski et al., 1996). 


\section{Table 3 (on next page)}

Conservation of amino acids within the interface between RRM3-RRM4 in PTB, nPTB and hnRNP-L 
Table 3: Conservation of amino acids within the interface between RRM3-RRM4 in PTB, nPTB and hnRNP-L

$\begin{array}{cccc}\text { RRM3 } & \text { PTB } & \text { nPTB } & \text { hnRNP-L } \\ & & & \\ & \text { S353 } & \text { S354 } & \text { R398 } \\ & \text { I356 } & \text { T357 } & \text { N401 } \\ \text { L357 } & \text { L358 } & \text { V402 } \\ & \text { V360 } & \text { V361 } & \text { L405 } \\ & \text { Y361 } & \text { Y362 } & \text { Y406 } \\ & \text { H397 } & \text { Q398 } & \text { N442 } \\ & \text { L399 } & \text { M400 } & \text { M444 } \\ & \text { H400 } & \text { Y401 } & \text { F445 } \\ \text { RRM4 } & & & \\ & \text { V501 } & \text { V501 } & \text { K552 } \\ & \text { V505 } & \text { I505 } & \text { L556 } \\ & \text { L508 } & \text { L508 } & \text { L559 } \\ & \text { I509 } & \text { I509 } & \text { G560 } \\ & \text { F526 } & \text { F526 } & \text { F582 }\end{array}$




\section{Figure 1}

Crystal structure of nPTB34.

Figure 1: Crystal structure of nPTB34. (a) Domain structure of nPTB. Residue numbers for domain boundaries are given along the top; percentages below indicate the sequence identity with PTB within each defined domain or linker region. (b) $3 \mathrm{~F}_{\mathrm{o}}-2 \mathrm{~F}_{\mathrm{c}}$ electron density map contoured at $1 \sigma$ for the refined crystal structure of nPTB34 (shown in stick representation). Carbon atoms in RRM3, RRM4 and the inter-domain linker are coloured pink, light-blue and grey respectively, a colour-scheme that is maintained throughout for nPTB34; nitrogen and oxygen atoms are coloured blue and red respectively. (c) The overall fold of the crystal structure of nPTB34 showing secondary structure features. (d) Alignment of all eight chains within the crystal asymmetric unit. View rotated with respect to panel c showing good overall alignment of secondary structure elements but differences in the linker.

a

1

57

135

182

284

336

431452

\begin{tabular}{|cccccccc} 
& RRM1 & & RRM2 & & RRM3 & RRM4 \\
\hline $55 \%$ & $81 \%$ & $45 \%$ & $88 \%$ & $63 \%$ & $81 \%$ & $100 \%$ & $80 \%$
\end{tabular}
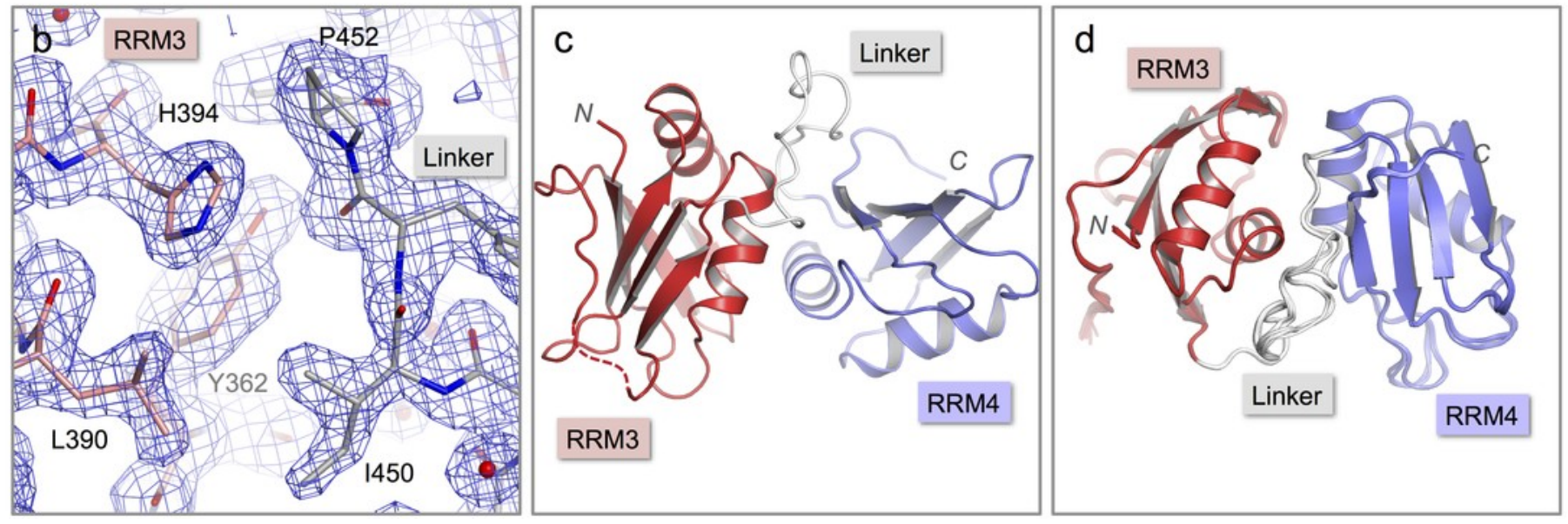


\section{Figure 2}

Solution structure of nPTB34 and comparison with related structures.

Figure 2: Solution structure of nPTB34 and comparison with related structures. (a) Ensemble of the 20 lowest energy solution structures of nPTB34 shown as $C_{\alpha}$ traces coloured by domain. (b) Ribbon representation of the superposition of the crystal structure of nPTB34 (coloured by domain) on the nPTB34 solution structure (yellow) (denoted by 'PX' and 'NMR' labels respectively. (c) Superposition of the crystal structure of nPTB34 (coloured by domain) on the solution structure of PTB34 (PDB ID 2evz; orange) (Vitali et al., 2006). (d) Superposition of the crystal structure of nPTB34 (coloured by domain) on the crystal structure of the equivalent domain from hnRNP-L (PDB ID 3to8; green) (Zhang et al., 2013). (e) Close up of the superposition shown in panel $d$ to illustrate the similarities and differences in the linker regions between RRMs 3 and 4 in nPTB34 and hnRNP-L.

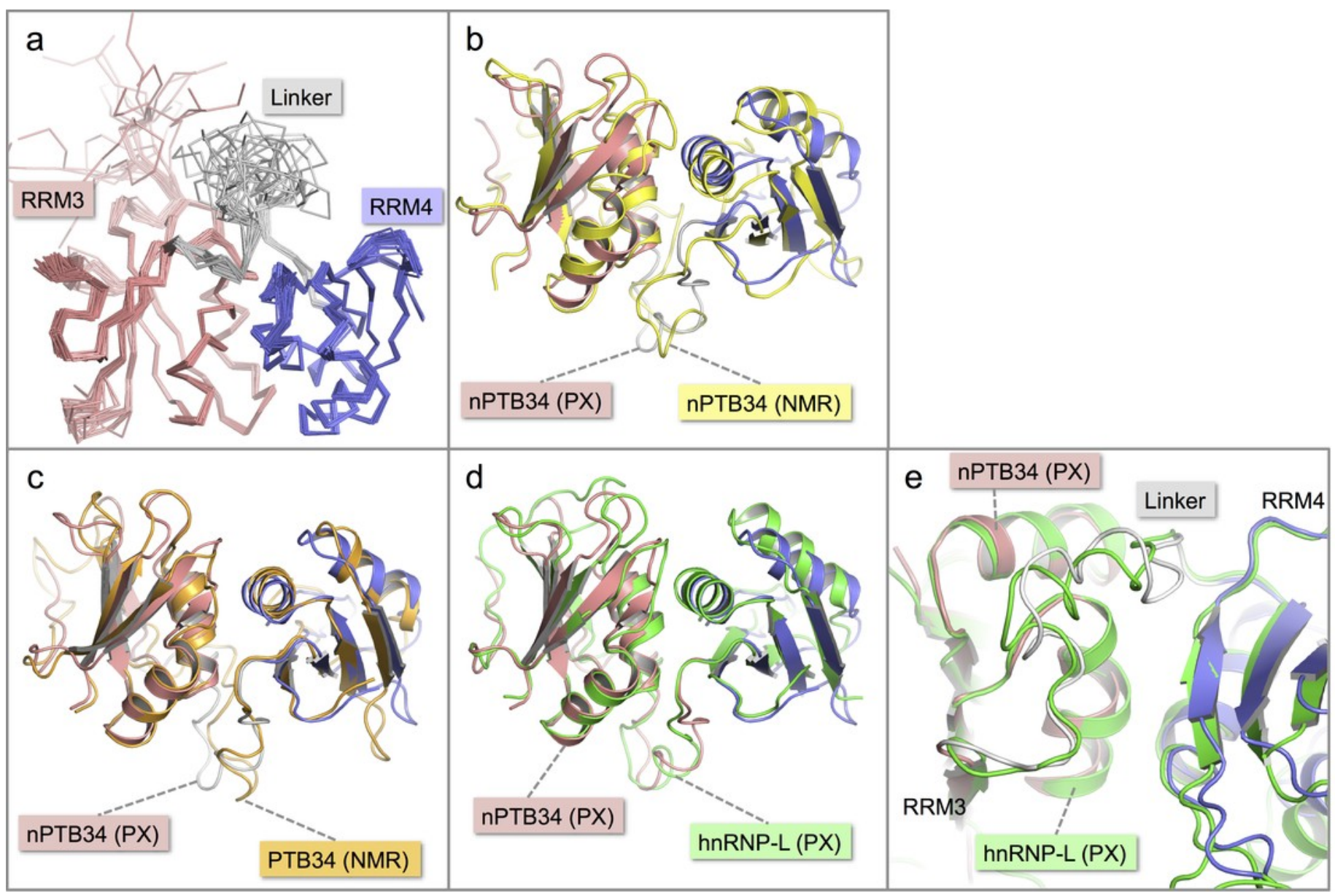




\section{Figure 3}

Comparison of the inter-domain interface within nPTB34 with the PTB RRM2-Raver1 interaction.

Figure 3: Comparison of the inter-domain interface within nPTB34 with the PTB RRM2-

Raver1 interaction. (a) The inter-domain interface in the crystal structure of nPTB34. RRM3 is shown with pink backbone and side-chain carbon atoms along with a semi-transparent rendering of its molecular surface (light-grey). The portion of RRM4 that contacts RRM3 within nPTB34 is show without its molecular surface. The oxygen atoms of bound water molecules located in the crystal structure are indicated by red spheres. (b) Superposition of the nPTB34 crystal structure (coloured as in panel a) with the structure of the PTB RRM2Raver1 complex (PDB ID 3zzy; RRM2 - green; Raver1 - yellow) (Joshi et al., 2011) .

Selected side chains are indicated. (c) The interaction between PTB RRM2 (green) and Raver1 (yellow). As for RRM3 in panel a, the molecular surface of RRM2 is indicated by a semi-transparent rendering.
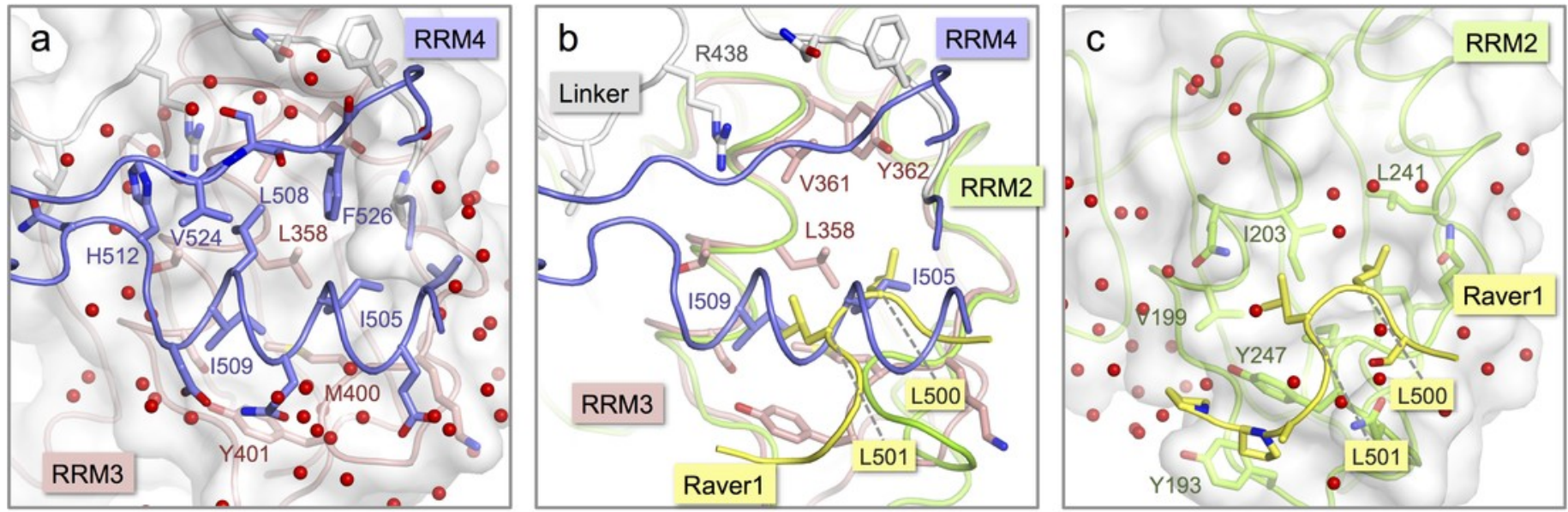


\section{Figure 4}

Location of amino acid differences on the RNA binding surfaces of PTB34 and nPTB34.

Figure 4: Location of amino acid differences on the RNA binding surfaces of PTB34 and nPTB34. (a)Superposition of the solution structure of RRM3 from PTB34 in complex with a hexameric CUCUCU RNA oligomer (PDB ID 2adc; grey) (Oberstrass et al., 2005) onto RRM3 from the crystal structure of nPTB34 (this work; pink). The disordered portion of the $\beta 4-\beta 5$ loop in RRM3 of nPTB34 is indicated by a dashed line. The side chains of amino acids that differ between the two structures (discussed in the text) are labeled to indicate the change from PTB34 to nPTB34 and shown for the solution structure of the PTB34-RNA complex. The structural difference in loop $\beta 2-\beta 3$ between the two structures shown most likely reflects conformational variability in this region in the absence of RNA. (b)

Superposition of the solution structure of RRM4 with the hexameric CUCUCU RNA oligomer from (PDB ID 2adc; grey) with RRM4 from the nPTB34 crystal structure (this work; lightblue). To avoid cluttering the figure only the four RNA nucleotides that make contact with RRM4 are shown. The side chains of amino acids that differ between the two structures are shown for the substitution R491H and the deletion of Lys 489 (PTB34) in nPTB34, which is labeled K491 $\Delta$.
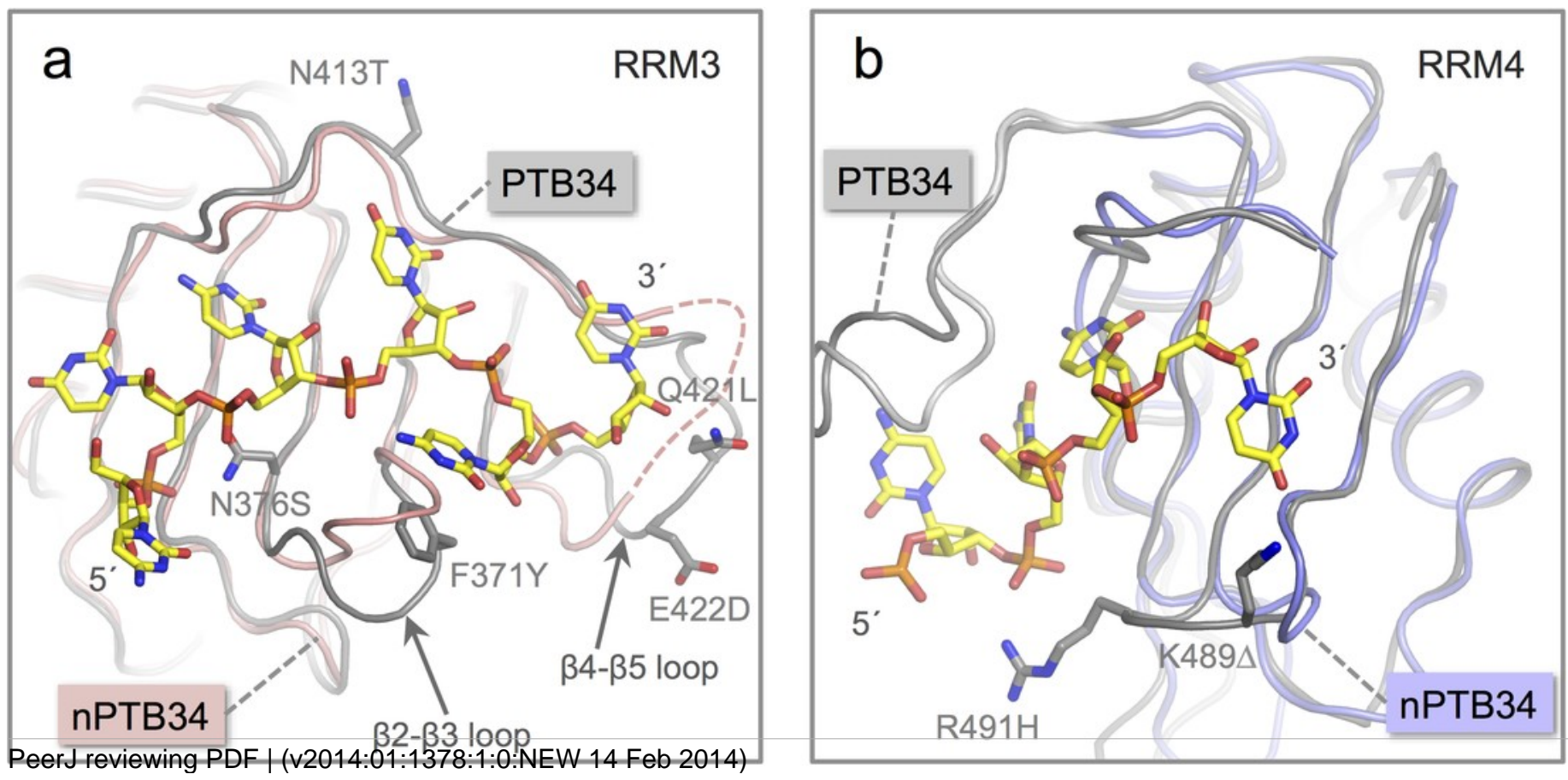